\title{
Energy transfer in a bilayer Fermi gas in the non-linear regime
}

\author{
B. Renklioglu, M. Ö. Oktel, and B. Tanatar* \\ Department of Physics, Bilkent University, 06800 Bilkent, Ankara, Turkey
}

Received 3 August 2016, revised 12 October 2016, accepted 24 October 2016

Published online 23 November 2016

Keywords energy transfer rate, dipolar interactions, drift velocity, nonlinear regime, random-phase approximation

*Corresponding author: e-mail tanatar@fen.bilkent.edu.tr, Phone: +90-312-2901591, Fax: +90-312-2664579

We consider a bilayer system of two-dimensional spin-polarized dipolar Fermi gas without any tunneling between the layers. We calculate the energy transfer rate between the layers in the nonlinear regime where the layers have a relative velocity, as a function of temperature and drift velocities of the particles of the system in each layer. The effective interactions describing the correlation effects and screening between the dipoles are obtained by the Hubbard approximation in a single layer (intralayer), and the random-phase approximation (RPA) across the layers (inter-layer). The energy transfer arises from the longrange nature of dipolar interactions between the particles of the system. As a result of the increasing drift velocities, the nonlinear heat transfer between the layers remarkably increases and the system reaches its equilibrium at lower temperatures. Our calculations show that cooling with dipolar interactions without any material contact can be utilized to cool the ultracold dipolar systems.
1 Introduction The properties of dipolar systems at low temperatures have attracted considerable attention in recent years after the realization of atomic species with magnetic dipole moments (e.g., chromium $(\mathrm{Cr})$ atoms [1-4], erbium (Er), atoms [5-8] and Dysprosium (Dy) atoms [8-11]) and successful studies on trapping and cooling of polar molecules with electrical dipole moments [12-16]. In these systems, the dipolar interaction dominates the system with its long-range and anisotropic character. The studies on dipolar systems reveal novel phases and previously unexplored regimes as a result of the unique nature of the dipolar interactions [17-21].

In most experimental studies on ultracold systems, the physical properties of the system are defined by the competition between the dipolar (long-range) and short-range interactions. The short-range interactions originate from the $\mathrm{S}$-wave scattering $[22,23]$. As a result of Feshbach resonance [1], the $S$-wave scattering length may be tuned to zero. Thus, the short-range interaction vanishes and a pure dipolar system appears $[3,4]$.

Generally, the cooling process of bosons depends on the short-range interactions because their thermalization is achieved by S-wave scattering. However, for spin-polarized Fermi gases, using spin mixtures or a mixture with another species is necessary in order to cool the system and this pro- cess is much more difficult. On the other hand, the anisotropic nature of the dipolar Bose or Fermi systems allows the mixing of different angular momentum channels. Depolarization cooling [24], demagnetization cooling [25], and spin distillation [26] are some techniques in which anisotropy and longranged behavior of the dipolar interaction are utilized.

In this paper, we consider the energy transfer phenomenon for a bilayer of purely dipolar spin-polarized Fermi system and investigate the effect of the dipolar interactions as a cooling scheme. The energy transfer is similar to the Coulomb drag effect which is a result of the momentum transfer between the layers, generated when an external field is applied to one of the layers. In the literature, the Coulomb drag effect as a transport method for electronic bilayer systems has extensively been reviewed [27-29]. When the transport properties do not depend on the external field (electric field in the case of charged systems, velocity field in the case of neutral systems) the bilayer system is said to be in the linear regime. We had earlier studied the energy transfer between dipolar Fermi gases in the linear regime [30]. Here, we focus on the non-linear regime in which the energy transfer depends on the flow velocities in the layers.

Energy transfer rate in bilayer systems depends on the temperature difference between the adjacent layers while there is no tunneling between them [31]. This energy 
transfer arises with (without) an external field applied to the system [32,33], and it is termed as the non-linear (linear) regime. The energy transfer was investigated for the double-layer graphene and double-quantum well (DQW) systems using the balance equation approach in the linear regime [34-36]. The results show that the obtained amount of transferred power for these systems is qualitatively similar to that achieved for the two-dimensional electron gas. In addition, non-linear energy transfer was investigated for the DQW systems through the balance equation approach $[37,38]$.

In our previous study, the intra-layer interactions were modeled by the Euler-Lagrange Fermi-hypernetted chain approximation (FHNC) [30]. In that work, we presented results for energy transfer in double layer dipolar ultracold Fermi gases in the linear regime at different temperatures and showed that this mechanism could be utilized as an effective cooling process due to the strong dipolar couplings [30]. Here, our calculations show that strong dipolar interactions might also be used as a cooling technique for ultracold polar molecules in the non-linear regime.

2 The model We investigate the energy transfer between two parallel layers of two-dimensional spin-polarized dipolar Fermi gas, separated by a distance $d$. We assume that there is no tunneling between the layers. The bare (unscreened) dipole-dipole interactions within a single layer $\mathcal{V}_{11}$ (intra-layer) and across the layers $\mathcal{V}_{12}$ (inter-layer) are given by

$$
\mathcal{V}_{11}(r)=\mathcal{V}_{22}(r)=\frac{C_{\mathrm{dd}}}{4 \pi} \frac{1}{r^{3}},
$$

and

$$
\mathcal{V}_{12}(r)=\frac{C_{\mathrm{dd}}}{4 \pi} \frac{r^{2}-2 d^{2}}{\left(r^{2}+d^{2}\right)^{5 / 2}},
$$

where the indices 1 and 2 show the corresponding layers and $r$ indicates the in-plane distance between dipoles. Here, $C_{\mathrm{dd}}$ indicates the dipole-dipole coupling constant, which is $C_{\mathrm{dd}}=p^{2} / \varepsilon_{0}$ for electric dipole moments $p$, and $C_{\mathrm{dd}}=\mu_{0} \mu^{2}$ for magnetic dipole moments $\mu$. In addition, $\mu_{0}$ is the vacuum permeability, $\varepsilon_{0}$ is the permittivity of free space.

The Fourier transform of the intra-layer interaction is $V_{11}(q)=V_{0}-C_{\mathrm{dd}} q / 2$ which includes a cut-off parameter $V_{0}$ related to the width of the layers $[33,39,40]$. Here, we adapt the Hubbard approximation for our calculations in order to obtain an effective intra-layer interaction in Fourier space without any cut-off parameter. For this purpose, a local-field factor $G_{\mathrm{H}}(q)=V_{11}\left(\sqrt{q^{2}+k_{\mathrm{F}}^{2}}\right) / V_{11}(q)$ is used in the sense $V_{11}(q) \rightarrow V_{11}(q)\left[1-G_{\mathrm{H}}(q)\right]$, yielding [41]

$$
V_{11}(q)=\frac{C_{\mathrm{dd}}}{2}\left[\sqrt{q^{2}+k_{\mathrm{F}}^{2}}-q\right] .
$$

Thus, our $V_{11}(q)$ contains intra-layer correlation effects to a certain degree. The approximation scheme we use here has been routinely employed in electronic systems [42]. For the inter-layer interaction, we use the Fourier transform of the bare interaction given by

$$
V_{12}(q)=-\frac{C_{\mathrm{dd}}}{2} q \exp (-q d) .
$$

The inter-layer interaction thus is treated at the level of random-phase approximation (RPA).

3 Energy transfer rate The rate of energy transfer as a result of interaction between two separate Fermi systems has been the subject of various investigations in recent years. These studies employed the balance equation approach [43], the quantum kinetic equation [44], and the non-equilibrium Green function method [31] for electron systems. Additionally, studies for one- and two-dimensional electron gases $[38,45]$ and graphene $[34,46]$ have been reported. In the present work, we mainly focus on neutral, ultracold dipolar gases, in contrast to the previous studies based on electronic systems.

The transferred power $\left(\mathcal{P}_{12}\right)$ per unit area from layer 2 to layer 1 can be calculated within the Fermi's Golden Rule by considering the energy transfer rate $[34,46]$ much like the case in drag effect where the momentum transfer rate [27] is calculated. Here, we use the Lei and Ting non-linear energy balance transport equation [46-49] as functions of layer temperatures $T_{i}$ and drift velocities $v_{i}$, given by

$$
\begin{aligned}
\mathcal{P}_{12}= & -\hbar \sum_{q} \int_{-\infty}^{\infty} \frac{\mathrm{d} \omega}{\pi} \omega\left|\frac{V_{12}(q)}{\epsilon\left(q, \omega, T_{1}, T_{2}\right)}\right|^{2} \\
& \times\left[n_{\mathrm{B}}\left(\frac{\hbar \omega}{k_{\mathrm{B}} T_{1}}\right)-n_{\mathrm{B}}\left(\frac{\hbar\left(\omega-\omega_{12}\right)}{k_{\mathrm{B}} T_{2}}\right)\right] \\
& \times \operatorname{Im} \chi_{1}\left(q, \omega, T_{1}\right) \operatorname{Im} \chi_{2}\left(q, \omega-\omega_{12}, T_{2}\right),
\end{aligned}
$$

where $\chi_{i}(q, \omega)$ is the finite temperature two-dimensional Lindhard polarization function [50] for the $i$ th layer and $\omega_{12}=q\left(v_{1}-v_{2}\right)$. The term $\omega-\omega_{12}$ appearing in $\chi_{2}$ may be regarded as the Galilean shift when an excitation $q$ is exchanged between two clouds with relative velocity. In the linear regime, the drift velocities are taken to be $v_{1}=$ $v_{2}=0$, whereas in the non-linear regime they are finite. Here, $n_{\mathrm{B}}(x)=1 /(\exp (x)-1)$ is the Bose-Einstein distribution function. In addition, the temperature dependent dielectric function $\epsilon\left(q, \omega, T_{1}, T_{2}\right)$ is given by the generalized random phase approximation [50] as

$$
\begin{aligned}
\epsilon\left(q, \omega, T_{1}, T_{2}\right)= & {\left[1-V_{11}(q) \chi_{1}\left(q, \omega, T_{1}\right)\right] } \\
& \times\left[1-V_{22}(q) \chi_{2}\left(q, \omega, T_{2}\right)\right] \\
& -\left[V_{12}(q)\right]^{2} \chi_{1}\left(q, \omega, T_{1}\right) \chi_{2}\left(q, \omega, T_{2}\right) .
\end{aligned}
$$

To characterize the system, we define two dimensionless parameters: (i) the dimensionless interaction strength $\lambda=a_{0} k_{\mathrm{F}}$ where $a_{0}$ indicates the characteristic length scale, 
obtained by $a_{0}=C_{\mathrm{dd}} m /\left(4 \pi \hbar^{2}\right)$. Here, $m$ is the mass of a dipole, $k_{\mathrm{F}}=\sqrt{4 \pi n}$ the Fermi wave number, $n$ is the $2 \mathrm{D}$ density of a single layer. (ii) The dimensionless separation distance $\widetilde{d}=d k_{\mathrm{F}}$, where $d$ is the distance between the layers.

In this work, the transferred power is calculated for the system in which the densities of the layers are equal $\left(n_{1}=n_{2}\right)$. In this symmetric case, dipolar gases confined to both layers of the system, have the same Fermi levels $\left(E_{\mathrm{F} 1}=E_{\mathrm{F} 2}=E_{\mathrm{F}}\right.$ and $\left.k_{\mathrm{F} 1}=k_{\mathrm{F} 2}=k_{\mathrm{F}}\right)$. As a result, the interaction strengths, the polarization functions and the effective potential interactions of the layers become equal to each other, as $\lambda_{1}=\lambda_{2}, \chi_{1}=\chi_{2}, V_{11}=V_{22}$, respectively.

4 Results The system in consideration consists of twodimensional spin-polarized dipolar Fermi gases separately confined into two parallel layers at which particles are driven by different drift velocities. In this study, we assume that particles in one of the layers (layer 1) with the temperature $T_{1}$, move with a drift velocity $v_{1}$. The other layer is supposed to be an open circuit, so $v_{2}=0$. We calculate the dimensionless energy transfer rate between the layers, separated by a distance $d$ at the non-linear regime. The dimensionless form of the energy transfer rate is defined as $P_{12}=\mathcal{P}_{12}\left[\hbar /\left(k_{\mathrm{F}} E_{\mathrm{F}}\right)^{2}\right]$.

The dimensionless energy transfer rate $\mathrm{P}_{12}$ between the layers is shown in Fig. 1 as function of $t_{2}=k_{\mathrm{B}} T_{2} / E_{F}$ for three different values of the drift velocities of the first layer $\left(\widetilde{v}_{1}=\left(\hbar k_{\mathrm{F}} / E_{\mathrm{F}}\right) v_{1}=0.0,0.5,1.0\right)$ while the temperature of the first layer is kept constant at Fermi value $T_{\mathrm{F}}$. The layer separation distance is $\widetilde{d}=1.0$ and the interaction strength is $\lambda=2.0$ in all of the calculations.

Energy is transferred from the hot layer to the cold one, so when the temperature $t_{2}$ surpasses $t_{1}$, the energy transfer rate $P_{12}$ changes sign. Consequently, at thermal equilibrium heat flow cannot be observed and the behavior of the energy transfer rate becomes linear in the temperature difference near this point, as shown in Fig. 1.

When the drift velocities are equal, so that $\omega_{12}=0$ in Eq. (6) (vanishingly small relative velocity between the layers), the energy transfer takes place in the linear regime. In this case, the thermal equilibrium occurs at $T_{1}=T_{2}$, as expected. The green-dotted line in Fig. 1 indicates the linear regime of the energy transfer in the system. As we increase the drift velocity in layer 1 , the interaction between the layers increases. Therefore, the equilibrium temperature occurs at lower temperatures.

We also investigate the drift velocity dependence of the energy transfer rate for different values of the interaction strength $\lambda$ while the distance between the layers is kept constant $(\widetilde{d}=1.0)$, as shown in Fig. 2. Here, the temperature of the layers is considered constant at $t_{1}=0.5$ and $t_{2}=1.0$. Once again, the second layer is assumed to be in an open circuit condition, $\widetilde{v}_{2}=0$. In Fig. 2 , as we increase the drift velocity of the first layer, the amount of transferred power increases.

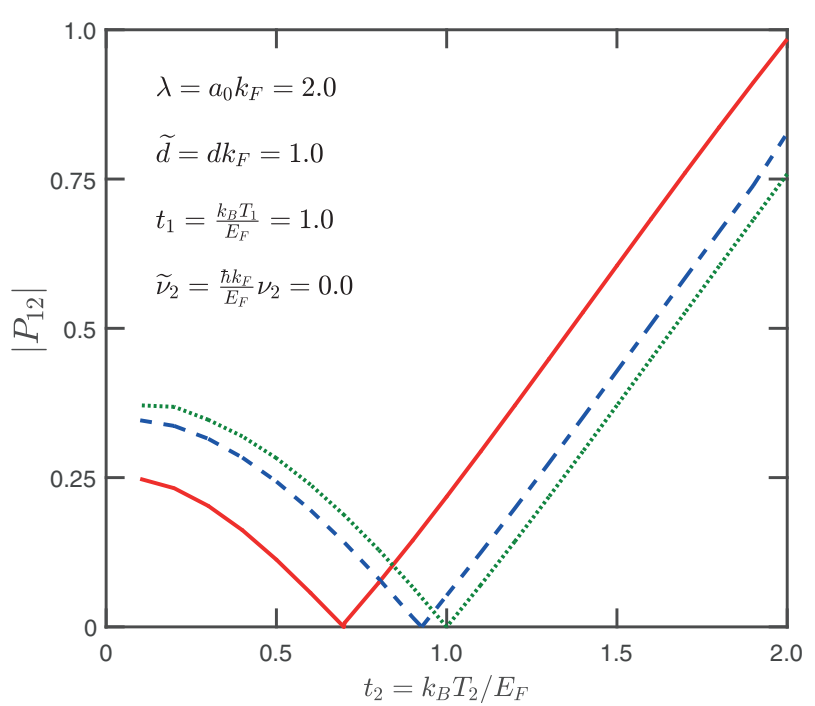

Figure 1 The absolute value of the dimensionless energy transfer rate $\left|P_{12}\right|$ as a function of the temperature $t_{2}$ of the second layer for three different values of drift velocities, $v_{1}$ of the Fermi gas confined in the first layer, indicated by green-dotted line (for $\widetilde{v}_{1}=$ 0 ), blue-dashed line (for $\widetilde{v}_{1}=0.5$ ), and red-solid line (for $\widetilde{v}_{1}=1.0$ ). The green-dotted line which indicates that the system has equal drift velocities $\widetilde{v}_{1}=\widetilde{v}_{2}=0$, corresponds to the linear regime of the energy transfer process. The second layer is assumed to be an open circuit, so we set $\widetilde{v}_{2}=0.0$. Here, the temperature of the first layer $t_{1}$ is kept constant at Fermi value. The dimensionless separation distance and the interaction strength parameter are considered as $\widetilde{d}=1.0$ and $\lambda=2$, respectively.

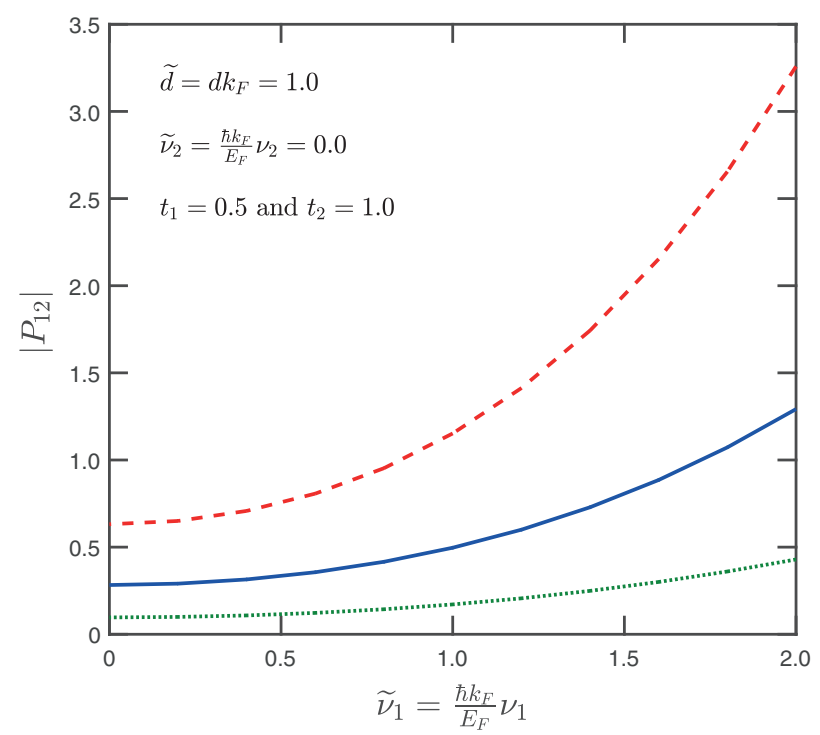

Figure 2 The absolute value of the dimensionless energy transfer rate $\left|P_{12}\right|$ as a function of the drift velocity $v_{1}$ of the first layer for three different values of interaction strength $\lambda$, indicated by greendotted line (for $\lambda=1.0$ ), blue-solid line (for $\lambda=2.0$ ), and reddashed line (for $\lambda=4.0$ ). Here, the separation distance between the layers and the drift velocity of the second layer are kept constant at $\widetilde{d}=1.0$ and $v_{2}=0.0$, respectively. The dimensionless temperatures of the layers are determined as $t_{1}=0.5$ and $t_{2}=1.0$. 
5 Conclusions In summary, we have calculated the transferred power between two parallel layers of twodimensional spin-polarized dipolar Fermi gases. We have shown that thermal equilibrium of the system may be reached at low temperatures as the drift velocity of one of the layers increases. In addition, the energy transfer rate also depends on the dipole-dipole interactions, and increases for larger values of the interaction strength, as expected.

Sympathetic cooling is achieved by the collisions between the components of two different gases in a mixture and this process terminates when the system reaches thermal equilibrium. Our calculations show that if the dipolar coupling is strong enough, it is not required to have a material contact between the components of the system. The dipolar coupling determines the heat transfer between the layers without taking into account the internal dynamics of each layer.

In order to demonstrate the feasibility of energy transfer in a bilayer as a cooling method for ultracold atoms and polar molecules, we examine three experimentally relevant parameters: (i) the length scale $a_{0}$; (ii) the density $n$; and (iii) the time constant $\tau$ for reaching thermal equilibrium. In our previous study, we expanded the energy transfer rate around the equilibrium point $\left(T_{1}=T_{2}\right)$ and linearized $P_{12}$ assuming a small temperature difference between the layers, i.e. $P_{12}=\kappa\left(T_{2}-T_{1}\right) / d$, where $\kappa$ is thermal conductivity [30]. The time constant is expressed as $\tau=d C_{V} / \kappa$, where $C_{V}$ is the heat capacity at constant volume of the 2D Fermi system [30].

Our calculations show that the length scales $a_{0}$ for some typical ultracold polar atoms with magnetic moments $\mu$ are much smaller than the size of the potential (around fewhundred nanometers) in the ultracold experiments. For instance, the length scales of the chromium $(\mathrm{Cr})$, erbium $(\mathrm{Er})$, and dysprosium (Dy) are calculated to be 2.4, 10.5, and $20.8 \mathrm{~nm}$, respectively. Therefore, the contactless sympathetic cooling method is not suitable for atomic systems with magnetic dipole moments.

On the other hand, as shown in Table 1, the ultracold polar molecules which require experimentally applicable length scales and moderate densities, have effective time constants

Table 1 Quantitative results for some ultracold polar molecules. Here, the interaction strength parameter and the dimensionless well separation distance are taken as $\widetilde{d}=1$ and $\lambda=2$, respectively. The estimated values of the time constant $\tau$ are obtained around the thermal equilibrium with $\left(t_{1} \simeq t_{2}\right)$ while one of the layers is assumed to be an open circuit, $v_{2}=0$. Note that $1 \mathrm{D}=3.34 \times 10^{-30} \mathrm{Cm}$.

\begin{tabular}{lllll}
\hline & $\mathrm{KRb}$ & $\mathrm{RbCs}$ & $\mathrm{NaK}$ & $\mathrm{LiCs}$ \\
\hline$p(\mathrm{D})$ & 0.57 & 1.3 & 2.72 & 5.5 \\
$a_{0}(\mu \mathrm{m})$ & 0.6 & 5.5 & 7.0 & 63.4 \\
$n\left(\mathrm{~m}^{-2}\right)$ & $8.84 \times 10^{11}$ & $1.05 \times 10^{10}$ & $6.50 \times 10^{9}$ & $7.92 \times 10^{7}$ \\
$\tau(\mathrm{ms}):$ & & & & \\
$\quad v_{1}=0.0$ & 0.126 & 18.52 & 8.53 & 1575 \\
$v_{1}=0.5$ & 0.117 & 17.20 & 8.00 & 1465 \\
$\nu_{1}=1.0$ & 0.088 & 13.01 & 6.00 & 1107 \\
\hline
\end{tabular}

which are smaller than the typical trap lifetimes. In addition, as the drift velocity of the first layer increases, a shorter time period will be required for the system to reach equilibrium which occurs at gradually decreasing temperatures, as presented in Table 1.

On the basis of the promising findings presented in this paper, the ultracold polar molecules can be sympathetically cooled without contact by utilizing strong dipolar interactions in the non-linear regime where the energy transfer takes place with an applied external field.

Acknowledgements We would like to thank the Scientific and Technological Research Council of Turkey (TÜBITAK) (Grant No. 116F030) for financial support. B.T. also thanks the Turkish Academy of Sciences (TUBA) for support.

\section{References}

[1] A. Griesmaier, J. Werner, S. Hensler, J. Stuhler, and T. Pfau, Phys. Rev. Lett. 94, 160401 (2005).

[2] J. Stuhler, A. Griesmaier, T. Koch, M. Fattori, T. Pfau, S. Giovanazzi, P. Pedri, and L. Santos, Phys. Rev. Lett. 95, 150406 (2005).

[3] T. Koch, T. Lahaye, J. Metz, B. Fröhlich, A. Griesmaier, and T. Pfau, Nature Phys. 4, 218 (2008).

[4] T. Lahaye, J. Metz, B. Fröhlich, T. Koch, M. Meister, A. Griesmaier, T. Pfau, H. Saito, Y. Kawaguchi, and M. Ueda, Phys. Rev. Lett. 101, 080401 (2008).

[5] J. J. McClelland and J. L. Hanssen, Phys. Rev. Lett. 96, 143005 (2006).

[6] K. Aikawa, A. Frisch, M. Mark, S. Baier, A. Rietzler, R. Grimm, and F. Ferlaino, Phys. Rev. Lett. 108, 210401 (2012).

[7] K. Aikawa, A. Frisch, M. Mark, S. Baier, R. Grimm, and F. Ferlaino, Phys. Rev. Lett. 112, 010404 (2014).

[8] T. Maier, H. Kadau, M. Schmitt, M. Wenzel, I. Ferrier-Barbut, T. Pfau, A. Frisch, S. Baier, K. Aikawa, L. Chomaz, M. J. Mark, F. Ferlaino, C. Makrides, E. Tiesinga, A. Petrov, and S. Kotochigova, Phys. Rev. X 5, 041029 (2015).

[9] M. Lu, N. Q. Burdick, and B. L. Lev, Phys. Rev. Lett. 108, 215301 (2012).

[10] M. Lu, N. Q. Burdick, S. H. Youn, and B. L. Lev, Phys. Rev. Lett. 107, 190401 (2011).

[11] T. Maier, H. Kadau, M. Schmitt, A. Griesmaier, and T. Pfau, Opt. Lett. 39, 3138 (2014).

[12] J. D. Weinstein, R. deCarvalho, T. Guillet, B. Friedrich, and J. M. Doyle, Nature 395, 148 (1998).

[13] H. L. Bethlem, G. Berden, F. M. H. Crompvoets, R. T. Jongma, A. J. A. van Roij, and G. Meijer, Nature 406, 491 (2000).

[14] J. M. Sage, S. Sainis, T. Bergeman, and D. DeMille, Phys. Rev. Lett. 94, 203001 (2005).

[15] S. Y. T. van de Meerakker, P. H. M. Smeets, N. Vanhaecke, R. T. Jongma, and G. Meijer, Phys. Rev. Lett. 94, 023004 (2005).

[16] J. R. Bochinski, E. R. Hudson, H. J. Lewandowski, and J. Ye, Phys. Rev. A 70, 043410 (2004).

[17] K. Góral, L. Santos, and M. Lewenstein, Phys. Rev. Lett. 88, 170406 (2002).

[18] N. R. Cooper and G. V. Shlyapnikov, Phys. Rev. Lett. 103, 155302 (2009).

[19] B. Capogrosso-Sansone, C. Trefzger, M. Lewenstein, P. Zoller, and G. Pupillo, Phys. Rev.Lett. 104, 125301 (2010). 
[20] L. Pollet, J. D. Picon, H. P. Büchler, and M. Troyer, Phys. Rev. Lett. 104, 125302 (2010).

[21] H. P. Büchler, E. Demler, M. Lukin, A. Micheli, N. Prokof'ev, G. Pupillo, and P. Zoller Phys. Rev. Lett. 98, 060404 (2007).

[22] S. Giorgini, L. P. Pitaevskii, and S. Stringari, Rev. Mod. Phys. 80, 1215 (2008).

[23] T. Lahaye, C. Menotti, L. Santos, M. Lewenstein, and T. Phau, Rep. Prog. Phys. 72, 126401 (2009).

[24] S. Hensler, A. Greiner, J. Stuhler, and T. Pfau, Europhys. Lett. 71, 918 (2005).

[25] M. Fattori, T. Koch, S. Goetz, A. Griesmaier, S. Hensler, J. Stuhler, and T. Pfau, Nature Phys. 2, 765 (2006).

[26] B. Naylor, E. Mar'echal, J. Huckans, O. Gorceix, P. Pedri, L. Vernac, and B. Laburthe-Tolra, Phys. Rev. Lett. 115, 243002 (2015).

[27] A. G. Rojo, J. Phys.: Condes. Matter 11, R31 (1999).

[28] R. Asgari, B. Tanatar, and B. Davoudi, Phys. Rev. B 77, 115301 (2008).

[29] B. N. Narozhny and A. Levchenko, Rev. Mod. Phys. 88, 025003 (2016).

[30] B. Renklioglu, B. Tanatar, and M. Ö. Oktel, Phys. Rev. A 93, 023620 (2016).

[31] X. F. Wang and I. C. da Cunha Lima, Phys. Rev. B 63, 205312 (2001).

[32] P. M. Solomon, P. J. Price, D. J. Frank, and D. C. La Tulipe, Phys. Rev. Lett. 63, 2508 (1989).

[33] E. H. Hwang and S. Das Sarma, Phys. Rev. B 84, 245441 (2011).
[34] B. Bahrami and T. Vazifehshenas, Phys. Lett. A 376, 3518 (2012).

[35] T. Vazifehshenas and T. Salavati-fard, Phys. Lett. B 462, 112 (2015).

[36] T. Vazifehshenas, B. Bahrami, and T. Salavati-fard, Phys. Lett. B 407, 4611 (2012).

[37] T. Salavati-fard and T. Vazifehshenas, Phys. Scr. 89, 125806 (2014).

[38] R. T. Senger and B. Tanatar, Solid State Commun. 121, 61 (2002).

[39] Q. Li, E. H. Hwang, and S. Das Sarma, Phys. Rev. B 82, 235126 (2010).

[40] N. T. Zinner and G. M. Bruun, Eur. Phys. J. D 65, 133 (2011).

[41] B. Tanatar, J. Low Temp. Phys. 171, 632 (2013).

[42] G. F. Giuliani and G. Vignale, Quantum Theory of the Electron Liquid (Cambridge University Press, Cambridge, 2005).

[43] H. L. Cui, X. L. Lei, and N. J. M. Horing, Superlattices Microstruct. 13, 221 (1993).

[44] I. I. Boiko, Yu. M. Sirenko, and P. Vasilopoulos, Phys. Rev. B 43, 7216 (1991).

[45] B. Tanatar, J. Appl. Phys. 81, 6214 (1997).

[46] M. Prunnila and S. J. Laakso, New J. Phys. 15, 033043 (2013).

[47] C. S. Ting, Physics of Hot Electron Transport in Semiconductors (World Scientific, Singapore, 1992).

[48] X. L. Lei and C. S. Ting, Phys. Rev. B 30, 4809 (1984).

[49] X. L. Lei and C. S. Ting, Phys. Rev. B 32, 1112 (1985).

[50] K. Flensberg and B. Y. Hu, Phys. Rev. B 52, 14796 (1995). 